\title{
Quantum theory within the probability calculus: a there-you-go theorem and partially exchangeable models
}

\author{
P.G.L. Porta Mana <pgleportamana.org>
}

6 March 2018

\begin{abstract}
"Ever since the advent of modern quantum mechanics in the late 1920's, the idea has been prevalent that the classical laws of probability cease, in some sense, to be valid in the new theory. [...] The primary object of this presentation is to show that the thesis in question is entirely without validity and is the product of a confused view of the laws of probability" (Koopman, 1957). The secondary objects are: to show that quantum inferences are cases of partially exchangeable statistical models with particular prior constraints; to wonder about such constraints; and to plead for a dialogue between quantum theory and the theory of exchangeable models.
\end{abstract}

\section{Introduction}

Ever since the advent of modern quantum mechanics in the late 1920's, the idea has been prevalent that the classical laws of probability cease, in some sense, to be valid in the new theory. More or less explicit statements to this effect have been made in large number and by many of the most eminent workers in the new physics [...]. Some authors have even gone farther and stated that the formal structure of logic must be altered to conform to the terms of reference of quantum physics [...].

Such a thesis is surprising, to say the least, to anyone holding more or less conventional views regarding the positions of logic, probability, and experimental science: many of us have been apt - perhaps too naively - to assume that experiments can lead to conclusions only when worked up by means of logic and probability, whose laws seem to be on a different level from those of physical science.

The primary object of this presentation is to show that the thesis in question is entirely without validity and is the product of a confused view of the laws of probability.

(B. O. Koopman, 1957) 
Koopman's lucid presentation stands today as it did sixty years ago. Perhaps it can be made even clearer if we consider quantum systems with a finite number of energy levels - qubits, qutrits, etc., very important in today's chase for quantum computers (Nielsen et al. 2010) - and if adopt the so-called "operational approach" to quantum theory, which can be glimpsed in Koopman's presentation itself.

\section{Operational approach}

A pseudohistorical presentation:

After decades of inconclusive debates about what quantum systems "really" are, the measurement problem, Schödinger's cats, and similar questions, the operational approach (see e.g.: Ludwig 1983; Segal 1959; Mielnik 1968; 1969; Lamb 1969; Davies et al. 1970; Foulis et al. 1972; Randall et al. 1973; 1979; Wright 1978; Haag 1982; Kraus 1983; Wootters 1986; Busch et al. 1995; for more recent elaborations: Hardy 2001; Porta Mana 2003; 2004a; Barnum et al. 2006; Barrett 2007; Harrigan et al. 2007) emerged as a way to sidestep or postpone answering them and get (blindly?) on with experiments and technology.

The starting points of this approach are these:

I. "all well-defined experimental evidence, even if it cannot be analysed in terms of classical physics, must be expressed in ordinary language making use of common logic [...]. This is a simple logical demand, since the word «experiment» can in essence only be used in referring to a situation where we can tell others what we have done and what we have learned" (Bohr 1948).

II. This verbalization of every experiment is conveniently divided into three parts: the descriptions of a preparation, of a measurement, and of several possible outcomes; each represented by a proposition: $S, M, O_{i}$. The outcomes, implicit in the description of the measurement, can be probabilistically predicted; including deterministic, unit-probability predictions. For each quantum system we have a set of possible preparations and a set of possible measurements; elements from the two sets can be freely combined, at least in principle.

III. To a preparation $S$ we can associate a unit-trace, positive-definite Hermitean matrix $\rho$ usually called density matrix; and to the outcomes $\left\{O_{i}\right\}$ of a measurement $M$, a set of positive-definite Hermitean 
matrices $\left\{\boldsymbol{E}_{i}\right\}$ summing up to the identity matrix; this set is called a positive-operator-valued measure. The dimension of these matrices depends on the system.

IV. The probability of obtaining outcome $O_{i}$ of the measurement $M$ when the preparation is $S$ is given by

$$
\mathrm{P}\left(O_{i} \mid M \wedge S\right)=\operatorname{tr} \boldsymbol{E}_{i} \rho,
$$

usually called the trace formula. The properties of the matrices guarantee that the probability distribution for the outcomes $\left\{O_{i}\right\}$ is non-negative and normalized.

In the verbalization of an experiment we can also include the description of a transformation, possibly parameterized by time; this is where Schrödinger's equation appears. Transformations are briefly discussed in appendix A. For the moment let's keep the description of this operational approach to a minimum. Appendix B shows how this approach comprises the old-fashioned quantum formalism with Hermitean operators \& Co.

The operational approach favours the view of probability as an extension of the propositional truth calculus (Keynes 1957; Johnson 1924; Ramsey 1926; Cox 1946; Pólya 1949; Jaynes 2003; Hailperin 1996; 2011; Terenin et al. 2017). Sure, we can translate all this in terms of "random variables" about physical quantities, but the verbal and propositional character of this approach is fundamental. It works because quantum physicists usually agree on the coarsest, protocol-like verbal description of an experiment, even if they may disagree on what is "really" going on microscopically. They agree on how to divide the experiment into preparation, measurement, and outcomes. They agree on which density matrices and positive-operator-valued measures to associate with those divisions. Each physicist can add his or her own personal interpretation $R_{\text {personal }}$ of what is "really" going on, but it becomes irrelevant when the coarse preparation is specified; we could write this irrelevance as

$$
\mathrm{P}\left(O_{i} \mid M \wedge S \wedge R_{\text {personal }}\right)=\mathrm{P}\left(O_{i} \mid M \wedge S\right) .
$$

The operational approach will thus still be valid if we'll eventually agree on a microscopic interpretation of quantum phenomena.

We shall now find additional reasons for the propositional view of probability in this approach. 


\section{Convexity of preparations}

The operational approach was accompanied by several developments in the mathematical formalism of quantum theory (e.g., the use of positiveoperator-valued measures), recruiting from subjects like $C^{*}$-algebras, lattice theory, convex spaces. The latter I find most insightful.

The set of positive-definite Hermitean matrices associated with preparations and measurement outcomes in points III-IV above can be seen as a subset of a real vector space of dimension $n^{2}$, where $n$ is the dimension of these matrices. The trace product in eq. (1) is just a scalar multiplication of such vectors (or better, the contraction of a vector and a dual 1-form, without scalar products). This means that we can associate a real-valued vector $s$ with each preparation, and a set of real-valued vectors $\left\{\boldsymbol{o}_{i}\right\}$ with each set of measurement outcomes, and the trace formula (1) becomes

$$
\mathrm{P}\left(O_{i} \mid M \wedge S\right)=\boldsymbol{o}_{i} \cdot s .
$$

A brilliant paper by Hardy (2001), foreshadowed by Wootters (1986), showed that this formula is true for any physical theory: quantum, classical, or otherwise. In fact, it holds for any collection of three kinds of propositions satisfying points I-II, whether they be about physical theories or not (Porta Mana 2003; 2004a).

The sets of vectors $\{\boldsymbol{s}\}$ and $\left\{\left\{\boldsymbol{o}_{i}\right\}\right\}$ satisfy constraints that guarantee the positivity and normalization of the probabilities; these constraints say that these sets are convex spaces. Classical and quantum systems differ in the convex properties of their sets of vectors $\{s\}$; let's call these states, and let's call extremal the preparations that are represented by extremal states of these convex sets. The set of states of a classical system is a simplex; that of a quantum system is the convex hull of complex projective space $\mathrm{CP}^{n-1}$ (Bengtsson et al. 2006). See figure on the right for an example with $n=3$ (qutrit). Because of these differing convex structures, for a classical system

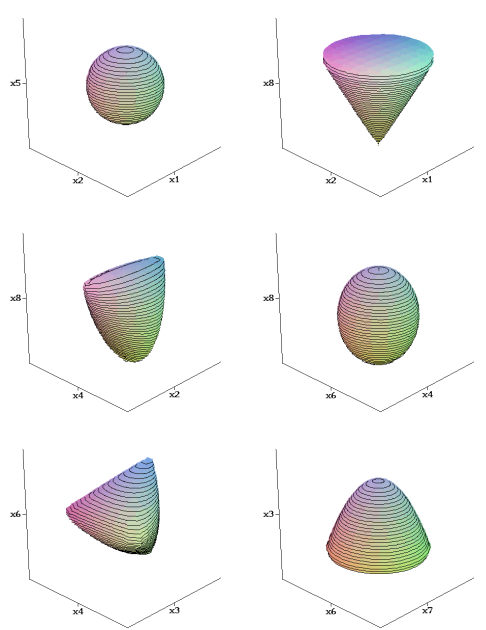

${ }_{3} \mathrm{D}$ sections of the convex hull of $\mathrm{CP}^{2}$ (Månsson et al. 2006) 
there is always a measurement that allows us to infer with probability 1 which of two extremal preparations was made:

there is $M$ such that $\mathrm{P}\left(S \mid M \wedge O_{i}\right)=0$ or 1 ,

$$
S \in\{\text { extremal preparations }\} ;
$$

whereas for a quantum system this is possible for particular extremal preparations only. These different behaviours under inference aren't foreign to the probability calculus, however. Kirkpatrick $(2003 \mathrm{a}, \mathrm{b})$ showed that analogous inferential characteristics appear in some games with cards, for example; similar examples are easily constructed with urndrawing (Porta Mana 2004b § IV).

Still today nobody knows why the sets of states of quantum systems have a projective-space convex structure. This is the "only mystery" (Feynman et al. 1965 § 1.1) of quantum theory.

In an experiment with a physical system - quantum or otherwise we can imagine a state of knowledge $S^{\prime}$ where we are unsure about which of two preparations $S_{1}, S_{2}$ was made, with corresponding probabilities:

$$
\mathrm{P}\left(S_{1} \mid S^{\prime}\right)=q_{1}, \quad \mathrm{P}\left(S_{2} \mid S^{\prime}\right)=q_{2}, \quad q_{1}+q_{2}=1 .
$$

In this state of knowledge our predictions for any measurement outcome will be, by the probability calculus,

$$
\begin{array}{r}
\mathrm{P}\left(O_{i} \mid M \wedge S^{\prime}\right)=\mathrm{P}\left(O_{i} \mid M \wedge S_{1}\right) \mathrm{P}\left(S_{1} \mid S^{\prime}\right)+\mathrm{P}\left(O_{i} \mid M \wedge S_{2}\right) \mathrm{P}\left(S_{2} \mid S^{\prime}\right)= \\
\left(\boldsymbol{o}_{i} \cdot \boldsymbol{s}_{1}\right) q_{1}+\left(\boldsymbol{o}_{i} \cdot \boldsymbol{s}_{2}\right) q_{2}=\boldsymbol{o}_{i} \cdot\left(q_{1} \boldsymbol{s}_{1}+q_{2} \boldsymbol{s}_{2}\right) .
\end{array}
$$

The first equality tacitly uses some logical-independence assumptions that are quite natural in an experimental setup; e.g., the choice of measurement doesn't tell us anything about the preparation.

The last equality says that we can associate the vector $q_{1} s_{1}+q_{2} s_{2}$, a convex combination of the states $s_{1}$ and $s_{2}$, with the state of knowledge $S^{\prime}$. This state of knowledge, usually called a mixture, can therefore be considered a citizen of the set of preparations.

It is natural to assume that states of knowledge like $S^{\prime}$ exist with all possible values of the distributions $\left(q_{1}, q_{2}\right)$, and also involving more than two preparations. This assumption implies that the "domain of discourse" for our system, even if it initially has only a finite number of preparations $\{S\}$ represented by states $\{s\}$, can always be extended to an infinite number of preparations, corresponding to the convex hull of $\{s\}$. 


\section{Lattice structure of measurements}

We can also consider two kinds of state of knowledge involving measurements and outcomes (cf. Peres et al. 1998).

The first, as with preparations, is a state of knowledge $M^{\prime}$ where we are unsure whether measurement $M_{1}$ or $M_{2}$ was made, with probabilities

$$
\mathrm{P}\left(M_{1} \mid M^{\prime}\right)=q_{1}, \quad \mathrm{P}\left(M_{2} \mid M^{\prime}\right)=q_{2}, \quad q_{1}+q_{2}=1 .
$$

The set of outcomes allowed by this state of knowledge is $\left\{O_{1 i}\right\} \cup\left\{O_{2 j}\right\}$, and we have

$$
\begin{array}{r}
\mathrm{P}\left(O_{1 i} \mid M^{\prime} \wedge S\right)=\mathrm{P}\left(O_{1 i} \mid M_{1} \wedge S\right) \mathrm{P}\left(M_{1} \mid M^{\prime}\right)+\mathrm{P}\left(O_{1 i} \mid M_{2} \wedge S\right) \mathrm{P}\left(M_{2} \mid M^{\prime}\right)= \\
\left(\boldsymbol{o}_{1 i} \cdot \boldsymbol{s}\right) q_{1}+0=\left(q_{1} \boldsymbol{o}_{1 i}\right) \cdot \boldsymbol{s}, \quad(8)
\end{array}
$$

and analogously for $\mathrm{O}_{2 j}$, assuming that the two original sets of outcomes are mutually exclusive. The state of knowledge $M^{\prime}$, usually called a mixture, can thus be considered a measurement, associated with the vectors $\left\{q_{1} \boldsymbol{o}_{1 i}\right\} \cup\left\{q_{2} \boldsymbol{o}_{2 j}\right\}$.

The second is a state of knowledge $M^{\prime \prime}$ in which we are not interested in the outcomes $\left\{O_{i}\right\}$ of a particular experiment $M$, but in other events $\left\{O_{j}^{\prime \prime}\right\}$ which we can probabilistically infer from those outcomes, with

$$
\mathrm{P}\left(O_{j}^{\prime \prime} \mid O_{i} \wedge M^{\prime \prime}\right)=Q_{j i}, \quad \sum_{j} Q_{j i}=1
$$

Then

$$
\begin{aligned}
\mathrm{P}\left(O_{j}^{\prime \prime} \mid M^{\prime \prime} \wedge S\right)=\sum_{i} \mathrm{P}\left(O_{j}^{\prime \prime} \mid O_{i} \wedge M^{\prime \prime}\right) \mathrm{P}\left(O_{i} \mid M \wedge S\right) & = \\
\sum_{i} Q_{j i}\left(\boldsymbol{o}_{i} \cdot \boldsymbol{s}\right) & =\left(\sum_{i} Q_{j i} \boldsymbol{o}_{i}\right) \cdot \boldsymbol{s},
\end{aligned}
$$

and we can consider $M^{\prime \prime}$ also as a measurement, associated with the vectors $\left\{\sum_{i} Q_{j i} \boldsymbol{o}_{i}\right\}$. We can call it a dither, but it includes coarsenings, i.e. situations where we aren't interested in distinguishing several outcomes; for example considering the set $\left\{\mathrm{O}_{1} \vee \mathrm{O}_{2}, \mathrm{O}_{3}\right\}$ instead of $\left\{\mathrm{O}_{1}, \mathrm{O}_{2}, \mathrm{O}_{3}\right\}$.

Mixtures and dithers can also be combined. They make the set of measurement outcomes into something more than a convex set: it is a set of lattices that can be combined in the two ways just described. 


\section{Conclusion}

"Conclusion?" you might ask. "Wasn't all the above just the preamble to a Proof that the standard probability calculus suffices for quantum theory? Where's the proof?" The proof was under your eyes as you were reading. The mathematical formalism just presented covers that of quantum systems with a finite number of energy levels, and with some topological care can be extended to cover systems with continuous energy levels, the Schrödinger equation, and even quantum field theory as infinite limits. This mathematical formalism is so general that it can also describe exotic systems that have neither classical nor quantum inferential traits. Did we have to generalize the ordinary probability calculus for this formalism? did we use any exotic probability theory? No. There you go.

Neither complex-valued probabilities nor lattices of $\sigma$-algebras have been necessary. In fact, what we've done looks simply like a special application of the probability calculus. The only special feature is the vector-product formula (3), expressing some probabilities as the results of vector products. But the probability calculus doesn't care where the numerical values of its probabilities come from, as long as they don't break its basic rules. And as we've seen, its rules are not broken when dealing with quantum experiments. Moreover, the vector-product formula doesn't have any physical significance: it appears when our domain of discourse involves three kinds of propositions logically related in a particular way (Porta Mana 2003; 2004a), but the propositions could be, say, about Donald Duck or parallel universes or other such things.

Only the particular convex structure of the preparations has physical significance, and it's experimentally observed. But the usual probability calculus can accommodate every convex structure, including the one peculiar to quantum theory.

The notion that quantum systems require lattices of $\sigma$-algebras - and therefore a generalization of the probability calculus - arises when we ignore the specification of the measurement in the conditional of the probabilities $\mathrm{P}\left(O_{i} \mid M \wedge S\right)$. We end up with several sets $\Omega_{M}$ and their $\sigma$-algebras, one for each $M$.

But this mathematical move does not make much sense, for at least three + one reasons. 
First, in real applications we often need to consider uncertainties about measurement procedures (Leonhardt 1997; de Muynck 2002 ch. 7; Ziman et al. 2006; D'Ariano et al. 2004). To do so we must use the mixing, dithering, coarsening formulae (7)-(10) within the standard probability calculus. In other words, we must gather the flock of separate sets $\Omega_{M}$ and $\sigma$-algebras back together, as subsets and subalgebras of one set only.

Second, the lattice structure of these $\sigma$-algebras reflects the operations of measurement mixing and dithering described in $\S 4$, operations clearly arising from conditionalization within one $\sigma$-algebra only.

Third, the game of wearing blinkers in order to see seemingly separate $\sigma$-algebras can be played in non-quantal, everyday contexts, like card or urn-drawing games (Kirkpatrick 2003a,b; Porta Mana 2004b § IV). Are these also "quantal"?

What's worse, this mathematical move inhibits an already nonexistent dialogue between quantum theory and the theory of statistical models based on exchangeability, sufficiency, symmetry (for a glimpse see Bernardo et al. 2000 ch. 4) that has been flourishing in probability and statistics since the 1930s, with many brilliant results and papers e.g. those by Koopman \& Pitman (Koopman 1936; Pitman 1936; Darmois 1935), Diaconis \& Freedman (Freedman 1962a,b; Diaconis 1977; 1988; 1992; Diaconis et al. 1980a,b,c; 1981; 1987; 1988; 1990), Martin-Löf (1974), Lauritzen (1974a,b; 1988; 1984; 2007), Ressel (1985), Aldous (1981; 1982; 1985; 2010), Kallenberg (1989; 2005), Cifarelli, Regazzini, Fortini, et al. (Cifarelli et al. 1979; 1980; 1981; 1982; Regazzini 1996; Fortini et al. 2000; 2002; 2012; 2014), to name very few besides those by de Finetti (1930; 1937; 1938), already known in the quantum literature. See Dawid's review (2013) for a small glimpse. Such a dialogue would surely benefit both disciplines, as I hope the ideas presented in the next section show.

\section{Quantum theory as a partially exchangeable model}

Many inferences in physics are instances of infinitely exchangeable statistical models (Bernardo et al. $2000 \S \S 4.2-3$ ):

$$
\mathrm{p}\left(D^{(1)}, D^{(2)}, \ldots \mid H\right)=\int\left[\prod_{i} \mathrm{p}\left(D^{(i)} \mid \theta, H\right)\right] \mathrm{p}(\theta \mid H) \mathrm{d} \theta,
$$

where $D^{(i)} \in\left\{O_{j}\right\}$ are observed outcomes of a set of experiments (1), (2), ... made in identical conditions and each $\mathrm{p}\left(D^{(i)} \mid \theta, H\right)$ is a 
categorical distribution (i.e. one-trial multinomial). This expression can be interpreted as a mixture of product probabilities $\mathrm{p}\left(D^{(i)} \mid \theta, H\right)$ indexed by the vector parameter $\theta$, weighted by the distribution $\mathrm{p}(\theta \mid H)$. The integration is defined over a simplex, but the distribution $\mathrm{p}(\theta \mid H)$ can effectively restrict it to a subset thereof. The distribution on the left side is usually called the predictive distribution.

The integral formula above results automatically when we assume that the joint probability of any number of outcomes is invariant under their permutations, no matter how many outcomes we consider. This assumption is called infinite exchangeability, and this result is de Finetti's representation theorem (1930; Hewitt et al. 1955). The theorem leaves undetermined the distribution $\mathrm{p}(\theta \mid H)$ only, usually called the prior. All infinitely exchangeable distributions over the outcomes are in one-one correspondence with all distributions $\mathrm{p}(\theta \mid H)$.

The exchangeability assumption can in turn be motivated by the identical condition in which the experiments were made. The remarkable part of this representation is that it automatically introduces mathematical objects analogous to the statistical states (Liouville distributions) $\{s\}$ of a discrete classical system: $\{\theta\} \equiv\{s\}$. We can interpret it as saying that each experiment was independently made with the same - but unknown - preparation $S$. Hence the integral, with the prior $\mathrm{p}(\theta \mid H) \equiv \mathrm{p}(s \mid H)$ representing our knowledge $H$ about the preparation. The features of this prior, like its support and maxima, may thus be motivated by physical laws or constraints.

Many authors (see the list at the end of $\S 5$ ) later proved various generalizations of this representation theorem, extending it to predictive distributions invariant under other symmetry groups, or possessing sufficient statistics.

Inference for quantum systems does not quite fit within the simple statistical model above, however. As we saw in the previous sections, quantum systems allow for a set $\{M\}$ of distinct measurements that cannot be obtained by marginalization from one another. Inferences for such systems therefore require that the conditional of the predictive distribution above specify which measurements $M^{(i)} \in\{M\}$ are performed, as shown in the probabilities of the previous sections. If we again interpret these experiments as independently made with the same but unknown preparation, we arrive (Porta Mana et al. 2006) at the 
expression

$$
\begin{aligned}
& \mathrm{p}\left(D^{(1)}, D^{(2)}, \ldots \mid M^{(1)}, M^{(2)}, \ldots, H\right)= \\
& \quad \quad \int_{\operatorname{conv} \mathbf{C P}{ }^{n-1}}\left[\prod_{i} \mathrm{p}\left(D^{(i)} \mid M^{(i)}, s, H\right)\right] \mathrm{p}(\boldsymbol{s} \mid H) \mathrm{d} s .
\end{aligned}
$$

The integration is over the convex hull of complex projective space $\mathrm{CP}^{n-1}$ (Bengtsson et al. 2006), as explained in $\S 3$, where $n$ is the number of quantum states completely distinguishable with a single measurement.

The expression above is a particular case of a partially exchangeable model (Bernardo et al. $2000 \S \S 4.6$; Gelman et al. 2014 ch. 5). The assumption of partial exchangeability states that the predictive distribution is invariant under permutations of outcomes of the same kind of measurement, but not across different kinds of measurement. This makes sense also because different measurements may have different numbers of outcomes. For examples of when and why this kind of assumption arises see Bernardo et al. (2000 ch. 4).

The assumption of partial exchangeability leads to a representation theorem too (de Finetti 1938; Bruno 1964; Diaconis et al. 1980b), of the form

$$
\begin{aligned}
& \mathrm{p}\left(D^{(1)}, D^{(2)}, \ldots \mid M^{(1)}, M^{(2)}, \ldots, H\right)= \\
& \quad \int\left[\prod_{i} \mathrm{p}\left(D^{(i)} \mid M^{(i)}, \eta_{M^{(i)}}, H\right)\right] \mathrm{p}\left[\left(\eta_{M}\right) \mid H\right] \prod_{M} \mathrm{~d} \eta_{M},
\end{aligned}
$$

where each outcome $D^{(i)}$ belongs to the set of possible outcomes of measurement $M^{(i)}$, these measurements belong to the system's set of possible measurements, $M^{(i)} \in\{M\}$, and each $\mathrm{p}\left(D^{(i)} \mid M^{(i)}, \eta_{M^{(i)}}, H\right)$ is a categorical distribution. Just like the expression (11) for infinite exchangeability, also this expression is a mixture of products of distributions indexed by parameters $\left(\eta_{M}\right)$, one for each kind of measurement, weighted by the prior $\mathrm{p}\left[\left(\eta_{M}\right) \mid H\right]$. The integration is defined over the Cartesian product of simplices $\prod_{M}\left\{\left(\eta_{M}\right)\right\}$, but the prior can effectively restrict it to a subset thereof. There is again a one-one correspondence between all partially exchangeable predictive distributions (left side) and all priors. The expression (11) for infinite exchangeability is a special case of the one above when $\{M\}$ comprises only one kind of measurement.

This generalized representation theorem is remarkable because it also automatically introduces a mathematical object, the parameter space 
$\left\{\left(\eta_{M}\right)\right\}$, which is similar to a space of states. We can interpret it as saying that each experiment was independently made with the same - but unknown - preparation $\left(\eta_{M}\right)$. Also in this case the prior $\mathrm{p}\left[\left(\eta_{M}\right) \mid H\right]$ expresses our knowledge $H$ about the possible preparations, and its functional features can be motivated by physical laws or constraints.

We said that the inferential formula (12) for quantum systems is a particular case of the expression (13) for partial exchangeability. Let's see what additional features make it a particular case. First we have to identify the states $s$ in the former with the parameters $\left(\eta_{M}\right)$ in the latter. Then we see that it is a particular case because the support of the prior $\mathrm{p}\left[\left(\eta_{M}\right) \mid H\right]$ is restricted to a particular lower-dimensional convex subset of the Cartesian product: the convex hull of a complex projective space. This restriction reflects the statistical properties of quantum systems, and is remarkably strong, because it reduces the support of the prior distribution from an infinite-dimensional manifold to a finite-dimensional one, for example from the function space $\left\{f \mid f: \mathbf{R P}^{2} \rightarrow[0,1]\right\}$ to the three-dimensional ball $\mathrm{CP}^{1}$ in the case of a qubit.

The full partially exchangeable model (13) allows for more general cases: less constrained than the quantum case, e.g. where each measurement has an outcome having probability 1; and more constrained than the quantum case, e.g. where no measurement can ever have a sure outcome.

For a quantum system, the a priori restriction on the prior of the partially exchangeable model reflects our empirical observation of measurement-outcome constraints typical of such systems: uncertainty relations, etc.. For example, if preparing an electron spin in a particular way we have probability 1 of obtaining $+z$ in a measurement along the $z$ direction, then with the same preparation we cannot have probability 1 or 0 of obtaining $+x$ in a measurement along the $x$ direction. We still don't know why such constraints exist. The mathematical formalism of quantum theory expresses and enforces these constraints, but doesn't explain why they exist either; just like the equation $\mathrm{d} S / \mathrm{d} t \geqslant 0$ (Truesdell 1984; Owen 1984) expresses and enforces the empirically found second law of thermodynamics, but doesn't explain why it must be so.

The exchangeability representation theorems (11) and (13) are welcome by many scientists, including yours faithfully, because they pull the notion of state out of the hat, thus also demoting it to a secondary, in principle avoidable notion. And they do so by promoting the notion 
of repeated, reproducible experiments, which science indeed hinges on. This point of view has been forcefully promoted by some probabilists in recent years (see e.g. Cifarelli et al. 1982; Regazzini 1996; Fortini et al. 2000).

The partially exchangeable model, including quantum inferences as special cases, thus demotes quantum states too. But it does so without de-emphasizing the physical and inferential properties characteristic of quantum systems, properties still reflected in the peculiar constraints of the model's prior. And moreover it emphasizes the necessity of considering several distinct measurements when dealing with quantum systems. These emphases should be contrasted with the features of the "quantum" exchangeability representation theorem by Hudson et al. (Hudson et al. 1976; Hudson 1981), neatened by Caves et al. (2002). This representation is surely useful in applications (van Enk et al. 2002; Fuchs et al. 2004). But it's tailor-made for quantum systems and therefore partially veils their peculiarity, if only by not openly showing the whole infinite-dimensional space of "unknown states" allowed by the full model; and it also veils the fact that quantum inferences need an assumption of partial exchangeability.

In the theory of exchangeable models it is known (Lauritzen 1988; Ressel 1985; Diaconis 1988; 1992; Kallenberg 2005; Dawid 2013) that the symmetries of a predictive distribution imply a particular form of its likelihood and the space of parameters - hence the support of the prior in the integral representation. This leads to interesting questions...

... for quantum theory: What kinds of symmetries or physical laws could cause the particular restrictions on the prior of the partially exchangeable model? The quantum literature offers studies of possible such symmetries (Wigner 1959; Haag 1996; Holevo 2011), but their discussion disregards partially exchangeable models.

... for the theory of exchangeable models: In which other contexts can or do analogous restrictions on the prior appear? Say, finance? biology? In which other contexts could they fruitfully be employed?

"Consent with both theories that they may enjoy each other: it shall be to our good" (Shakespeare 1623 scene V.II, paraphrased). 


\section{Thanks}

... to H. Barnum, A. Terenin, D. Draper for unintentionally rekindling my interest in these matters. To Mari \& Miri for continuous encouragement and affection. To Buster Keaton for filling life with awe. To the developers and maintainers of LATEX, Emacs, AUCTEX, Open Science Framework, biorXiv, Hal archives, Python, Inkscape, Sci-Hub for making a free and unfiltered scientific exchange possible.

\section{Appendices}

\section{A Transformations}

In the operational approach, the transformation of a preparation is some knowledge $T$, possibly dependent on a parameter like time (hence a collection of such propositions), that allows us to infer one preparation from another, possibly at different times, and possibly across different physical systems:

$$
\mathrm{P}(\hat{S} \mid T \wedge S)=q .
$$

This notion can be shown to subsume the usual notions of deterministic evolution, stochastic evolution, and collapse, and can be naturally combined with all the probabilistic formulae we have seen so far. Every transformation can be associated with a linear map acting on the state vectors: $\hat{s}=T s$. We can also consider mixtures of transformations, and so on.

It's important to note that Schrödinger's equation describes the timedependence of the linear map $\boldsymbol{T}$ associated with a particular transformation $T$ - not of the probability (14) itself.

\section{B Traditional quantum theory from the operational approach}

In the space of such matrices of positive-definite Hermitean matrices of dimension $n$ we can always find $n$ orthogonal projectors $\left\{\boldsymbol{\Pi}_{i}\right\}$ such that $\boldsymbol{\Pi}_{i} \boldsymbol{\Pi}_{j}=\delta_{i j} \boldsymbol{\Pi}_{i}$. Such projectors have unit trace; they can thus represent the density matrix associated with a preparation. They can also be written as $\left|\psi_{i}\right\rangle\left\langle\psi_{i}\right|$, where $\left\langle\psi_{i}\right|$ is a unit complex vector and $\left|\psi_{i}\right\rangle$ its dual. The sum of orthogonal projectors is the identity matrix; a set of orthogonal projectors can thus be associated with the outcomes of a measurement, too. 
When we consider a preparation and a measurement outcome associated with orthogonal projectors $E_{k}, E_{i}$, the trace formula becomes the famous

$$
\mathrm{P}\left(O_{i} \mid M \wedge S\right)=\operatorname{tr} E_{i} E_{k} \equiv\left|\left\langle\psi_{i} \| \psi_{k}\right\rangle\right|^{2} .
$$

If the outcomes $\left\{O_{i}\right\}$ of a measurement associated with orthogonal projectors are numerical values $\left\{\lambda_{i}\right\}$, the expected value is another famous formula:

$$
\sum_{i} \lambda_{i} \mathrm{P}\left(O_{i} \mid M \wedge S\right)=\sum_{i} \lambda_{i} \operatorname{tr} \boldsymbol{E}_{i} \rho=\left\langle\psi_{k}\left|\left[\sum_{i} \lambda_{i}\left|\psi_{i}\right\rangle\left\langle\psi_{i}\right|\right]\right| \psi_{k}\right\rangle,
$$

where the expression in brackets is a Hermitean operator with real spectrum.

Orthogonal projector matrices are associated with preparations and measurements which jointly lead to completely certain outcomes, in the sense of eq. (4). The more general density matrices and positiveoperator-valued measures were introduced to describe experimental situations in which noise sources make the preparation uncertain, and interaction with other systems during measurement can lead to noise in the outcomes or even to their proliferation (Busch et al. 1989; de Muynck 2002 ch. 7). The more general measurements associated with positiveoperator-valued measures also include simultaneous measurements of conjugate quantities like position and momentum (Arthurs et al. 1965; Busch et al. 1984; Appleby 1998), which are routine in fields like quantum optics (Leonhardt 1997 ch. 6).

\section{Bibliography}

("de X" is listed under $\mathrm{D}$, "van $X$ " under $\mathrm{V}$, and so on, regardless of national conventions.)

Aldous, D. J. (1981): Representations for partially exchangeable arrays of random variables. J. Multivariate Anal. 114, $581-598$.

- (1982): On exchangeability and conditional independence. In: Koch, Spizzichino (1982), 165-170.

- (1985): Exchangeability and related topics. In: Aldous, Ibragimov, Jacod (1985), VII, 1-198. https://Www. stat. berkeley. edu/ aldous/206-Exch/Papers/papers.html.

- (2010): More uses of exchangeability: representations of complex random structures. In: Bingham, Goldie (2010), ch. 2, 35-63.

Aldous, D. J., Ibragimov, I. A., Jacod, J. (1985): École d'Été de Probabilités de Saint-Flour XIII 1983. (Springer, Berlin). Édité par P. L. Hennequin.

Appleby, D. M. (1998): Concept of experimental accuracy and simultaneous measurements of position and momentum. Int. J. Theor. Phys. $37^{5}, 1491-1509$. 
Arthurs, E., Kelly Jr., J. L. (1965): On the simultaneous measurement of a pair of conjugate observables. Bell Syst. Tech. J. 44 ${ }^{4}, 725-729$.

Barndorff-Nielsen, O. E., Blæsild, P., Schou, G., eds. (1974): Proceedings of Conference on Foundational Questions in Statistical Inference: Aarhus, May 7-12, 1973. (University of Aarhus, Aarhus).

Barndorff-Nielsen, O. E., Dawid, A. P., Diaconis, P., Johansen, S., Lauritzen, S. L. (1984): Discussion of Steffen Lauritzen's paper ["Extreme Point Models in Statistics"]. Scand. J. Statist. 11 ${ }^{2}, 83-91$. See Lauritzen (1984).

Barnum, H., Barrett, J., Leifer, M., Wilce, A. (2006): Cloning and broadcasting in generic probabilistic theories. arXiv: quant-ph/0611295.

Barrett, J. (2007): Information processing in generalized probabilistic theories. Phys. Rev. A 753, 032304 .

Bengtsson, I., Życzkowski, K. (2006): Geometry of Quantum States: An Introduction to Quantum Entanglement. (Cambridge University Press, Cambridge).

Bernardo, J.-M., DeGroot, M. H., Lindley, D. V., Smith, A. F. M., eds. (1988): Bayesian Statistics 3. (Oxford University Press, Oxford).

Bernardo, J.-M., Smith, A. F. (2000): Bayesian Theory, reprint. (Wiley, New York). First publ. 1994.

Bingham, N. H., Goldie, C. M., eds. (2010): Probability and Mathematical Genetics: Papers in Honour of Sir John Kingman. (Cambridge University Press, Cambridge).

Bohr, N. (1948): On the notions of causality and complementarity. Dialectica $\mathbf{2}^{3-4}, 312-319$.

Browder, F. E., ed. (1992): Mathematics into the Twenty-first Century: 1988 Centennial Symposium August 8-12. (American Mathematical Society, Providence, USA).

Bruno, A. (1964): On the notion of partial exchangeability. Giorn. Ist. Ital. Att. 27, 174-196. Transl. in de Finetti (1972), ch. 10, pp. 229-246.

Busch, P., Grabowski, M., Lahti, P. J. (1989): Some remarks on effects, operations, and unsharp measurements. Found. Phys. Lett. 24, 331-345.

- (1995): Operational Quantum Physics. (Springer, Berlin).

Busch, P., Lahti, P. J. (1984): On various joint measurements of position and momentum observables in quantum theory. Phys. Rev. D 29 ${ }^{8}, 1634-1646$.

Caves, C. M., Fuchs, C. A., Schack, R. (2002): Unknown quantum states: the quantum de Finetti representation. J. Math. Phys. 43 ${ }^{9}, 4537-4559$.

Cifarelli, D. M., Regazzini, E. (1979): Considerazioni generali sull'impostazione bayesiana di problemi non parametrici. Le medie associative nel contesto del processo aleatorio di Dirichlet. Riv. mat. scienze econ. sociali $\mathbf{2}^{1,2}, 39-52,95^{-111 .}$

- (1980): Sul ruolo dei riassunti esaustivi ai fini della previsione in contesto bayesiano ( $1^{a}$ parte). Riv. mat. scienze econ. sociali $3^{2}, 109-125$. See also Cifarelli, Regazzini (1981).

- (1981): Sul ruolo dei riassunti esaustivi ai fini della previsione in contesto bayesiano ( $2^{a}$ parte). Riv. mat. scienze econ. sociali $4^{1}, 3^{-11}$. See also Cifarelli, Regazzini (1980).

- (1982): Some considerations about mathematical statistics teaching methodology suggested by the concept of exchangeability. In: Koch, Spizzichino (1982), 185-205.

Cox, R. T. (1946): Probability, frequency, and reasonable expectation. Am. J. Phys. 14 ${ }^{1}, 1-13$. http://algomagic.org/ProbabilityFrequencyReasonableExpectation.pdf.

Damien, P., Dellaportas, P., Polson, N. G., Stephens, D. A., eds. (2013): Bayesian Theory and Applications. (Oxford University Press, Oxford).

D’Ariano, G. M., Maccone, L., Lo Presti, P. (2004): Quantum calibration of measurement instrumentation. Phys. Rev. Lett. 93, 250407. 
Darmois, G. (1935): Sur les lois de probabilité à estimation exhaustive. Comptes rendus hebdomadaires des séances de l'Académie des sciences 200, 1265-1266.

Davies, E. B., Lewis, J. T. (1970): An operational approach to quantum probability. Commun. Math. Phys. $17^{3}, 239-260$.

Dawid, A. P. (2013): Exchangeability and its ramifications. In: Damien, Dellaportas, Polson, Stephens (2013), ch. 2, 19-29.

de Finetti, B. (1930): Funzione caratteristica di un fenomeno aleatorio. Atti Accad. Lincei: Sc. Fis. Mat. Nat. IV 5 , 86-133. http://www. brunodefinetti.it/Opere.htm.

- (1937): La prévision : ses lois logiques, ses sources subjectives. Ann. Inst. Henri Poincaré $7^{1}$, 1-68. Transl. in Kyburg, Smokler (1980), pp. 53-118, by Henry E. Kyburg, Jr.

- (1938): Sur la condition d'équivalence partielle. In: Colloque consacré à la théorie des probabilités. VI : Conceptions diverses. Ed. by B. de Finetti, V. Glivenko, G. Neymann (Hermann, Paris), 5-18. Transl. in Jeffrey (1980), pp. 193-205, by P. Benacerraf and R. Jeffrey.

- (1972): Probability, Induction and Statistics: The art of guessing. (Wiley, London).

de Muynck, W. M. (2002): Foundations of Quantum Mechanics, an Empiricist Approach. (Kluwer, Dordrecht).

Diaconis, P. (1977): Finite forms of de Finetti's theorem on exchangeability. Synthese $\mathbf{3 6}^{2}$, 271-281. http://statweb. stanford. edu/ cgates/PERSI/year. html.

- (1988): Recent progress on de Finetti's notions of exchangeability. In: Bernardo, DeGroot, Lindley, Smith (1988), 111-125. With discussion by D. Blackwell, Simon French, and author's reply. http: / / statweb. stanford. edu/ cgates / PERSI / year . html, https ://statistics . stanford. edu/research/recent - progress - de - finettis - notions - exchangeability.

- (1992): Sufficiency as statistical symmetry. In: Browder (1992), 15-26. First publ. 1991 as technical report https://statistics.stanford.edu/research/sufficiency-statis tical - symmetry.

Diaconis, P., Freedman, D. (1980a): Finite exchangeable sequences. Ann. Prob. 84, 745-764.

- (1980b): De Finetti's generalizations of exchangeability. In: Jeffrey (1980), 233-249.

- (1980c): De Finetti's theorem for Markov chains. Ann. Prob. 8 ${ }^{1}, 115-130$.

- (1981): Partial exchangeability and sufficiency. In: Ghosh, Roy (1981), 205-236. http://s tatweb. stanford. edu/ cgates/PERSI/year.html. Also publ. 1982 as technical report https://WWw.stat.berkeley.edu/ aldous/206-Exch/Papers/diaconis_freedman PES.pdf.

- (1987): A dozen de Finetti-style results in search of a theory. Ann. Inst. Henri Poincaré (B) $23^{\mathrm{S} 2}, 397-423$.

- (1988): Conditional limit theorems for exponential families and finite versions of de Finetti's theorem. J. Theor. Probab. 14, 381-410.

- (1990): Cauchy's equation and de Finetti's theorem. Scand. J. Statist. 173 , 235-249. http ://nma. berkeley.edu/ark:/28722/bk000471m2r.

Feynman, R. P., Leighton, R. B., Sands, M. (1965): The Feynman Lectures on Physics: Quantum Mechanics. (Addison-Wesley, Reading, USA).

Fortini, S., Ladelli, L., Petris, G., Regazzini, E. (2002): On mixtures of distributions of Markov chains. Stochastic Processes Appl. 100 ${ }^{1-2}, 147-165$.

Fortini, S., Ladelli, L., Regazzini, E. (2000): Exchangeability, predictive distributions and parametric models. Sankhyā A $\mathbf{6 2}^{1}, 86-109$. 
Fortini, S., Petrone, S. (2012): Predictive construction of priors in Bayesian nonparametrics. Braz. J. Probab. Stat. 264, 423-449. http://didattica. unibocconi.it/mypage/upload/490 4120150619104659 2012BRAZIL. PDF.

- (2014): Predictive characterization of mixtures of Markov chains. arXiv: 1406.5421.

Foulis, D. J., Randall, C. H. (1972): Operational statistics. I. Basic concepts. J. Math. Phys. 13 ${ }^{11}$, 1667-1675. See also Randall, Foulis (1973).

Freedman, D. A. (1962a): Mixtures of Markov processes. Ann. Math. Stat. $33^{1}, 114-118$.

- (1962b): Invariants under mixing which generalize de Finetti's theorem. Ann. Math. Stat. $33^{3}, 916-923$.

Fuchs, C. A., Schack, R., Scudo, P. F. (2004): De Finetti representation theorem for quantumprocess tomography. Phys. Rev. A 69, 062305.

Gelman, A., Carlin, J. B., Stern, H. S., Dunson, D. B., Vehtari, A., Rubin, D. B. (2014): Bayesian Data Analysis, 3rd ed. (Chapman \& Hall/CRC, Boca Raton, USA). First publ. 1995.

Ghosh, J. K., Roy, J., eds. (1981): Statistics: Applications and New Directions. (Indian Statistical Institute, Calcutta).

Haag, R. (1982): Mathematical structure of orthodox quantum theory and its relation to operationally definable physical principles. In: Schrader, Seiler, Uhlenbrock (1982), 168-172.

- (1996): Local Quantum Physics: Fields, Particles, Algebras, 2nd ed. (Springer, Berlin). First publ. 1992.

Hailperin, T. (1996): Sentential Probability Logic: Origins, Development, Current Status, and Technical Applications. (Associated University Presses, London).

- (2011): Logic with a Probability Semantics: Including Solutions to Some Philosophical Problems. (Lehigh University Press, Plymouth, UK).

Hardy, L. (2001): Quantum theory from five reasonable axioms. arXiv : quant - ph/0101012.

Harrigan, N., Rudolph, T., Aaronson, S. (2007): Representing probabilistic data via ontological models. arXiv:0709.1149.

Henkin, L., Suppes, P., Tarski, A., eds. (1959): The Axiomatic Method: With Special Reference to Geometry and Physics. (North-Holland, Amsterdam). http://www. archive.org/det ails/axiomaticmethod031862mbp.

Hewitt, E., Savage, L. J. (1955): Symmetric measures on Cartesian products. Trans. Am. Math. Soc. $80^{2}, 470-501$.

Holevo, A. S. (2011): Probabilistic and Statistical Aspects of Quantum Theory, 2nd ed. (Edizioni della Normale, Pisa). First publ. in Russian 1980.

Hooker, C. A., ed. (1979): Physical Theory as Logico-Operational Structure. (D. Reidel, Dordrecht).

Hudson, R. L. (1981): Analogs of de Finetti's theorem and interpretative problems of quantum mechanics. Found. Phys. 119-10, $805^{-808 . ~}$

Hudson, R. L., Moody, G. R. (1976): Locally normal symmetric states and an analogue of de Finetti's theorem. Z. Wahrscheinlichkeitstheorie verw. Gebiete $33^{4}, 343-351$.

Jaynes, E. T. (2003): Probability Theory: The Logic of Science. (Cambridge University Press, Cambridge). Ed. by G. Larry Bretthorst; http://www-biba.inrialpes.fr/Jaynes/p rob.html, http://omega.albany. edu:8008/JaynesBook.html, http://omega. al bany . edu: 8008/JaynesBookPdf . html. First publ. 1994.

Jeffrey, R. C., ed. (1980): Studies in inductive logic and probability. Vol. II. (University of California Press, Berkeley).

Johnson, W. E. (1924): Logic. Part III: The Logical Foundations of Science. (Cambridge University Press, Cambridge). https : //archive.org/details/logic03john, https ://archive.org/details/johnsonslogico3johnuoft. 
Kallenberg, O. (1989): On the representation theorem for exchangeable arrays. J. Multivariate Anal. $3^{1}, 137-154$.

- (2005): Probabilistic Symmetries and Invariance Principles. (Springer, New York).

Keynes, J. M. (1957): A Treatise on Probability. (Macmillan, London). http://www . archive .org/details/ATreatise0nProbability. First publ. 1921.

Khrennikov, A. Yu., ed. (2004): Quantum Theory: Reconsideration of Foundations - 2. (Växjö University Press, Växjö, Sweden).

Kirkpatrick, K. A. (2003a): "Quantal” behavior in classical probability. Found. Phys. Lett. 163, 199-224. First publ. 2001.

- (2003b): Classical three-box "paradox". J. Phys. A 36 ${ }^{17}, 4891-4900$. First publ. 2002. See also Ravon, Vaidman (2007) and Kirkpatrick (2007).

- (2007): Reply to 'The three-box paradox revisited' by T. Ravon and L. Vaidman. J. Phys. A $\mathbf{4 0}^{11}, 2883-2890$. See Kirkpatrick (2003b) and Ravon, Vaidman (2007).

Koch, G., Spizzichino, F., eds. (1982): Exchangeability in Probability and Statistics. (NorthHolland, Amsterdam).

Koopman, B. O. (1936): On distributions admitting a sufficient statistic. Trans. Am. Math. Soc. $39^{3}, 399-409$.

- (1957): Quantum theory and the foundations of probability. In: MacColl (1957), 97-102.

Kraus, K. (1983): States, Effects, and Operations: Fundamental Notions of Quantum Theory. (Springer, Berlin).

Kyburg Jr., H. E., Smokler, H. E., eds. (1980): Studies in Subjective Probability, 2nd ed. (Robert E. Krieger, Huntington, USA). First publ. 1964.

Lamb Jr., W. E. (1969): An operational interpretation of nonrelativistic quantum mechanics. Phys. Today $22^{4}, 23-28$.

Lauritzen, S. L. (1974a): Sufficiency, prediction and extreme models. Scand. J. Statist. $\mathbf{1}^{3}$, 128-134.

- (1974b): Sufficiency, prediction and extreme models. In: Barndorff-Nielsen, Blæsild, Schou (1974), 249-269. With discussion.

- (1984): Extreme point models in statistics. Scand. J. Statist. $\mathbf{1 1}^{2}, 65-83$. See also discussion Barndorff-Nielsen, Dawid, Diaconis, Johansen, Lauritzen (1984).

- (1988): Extremal Families and Systems of Sufficient Statistics. (Springer, Berlin). First publ. 1982.

- (2007): Sufficiency, partial exchangeability, and exponential families. http://www. stats.ox . ac. uk/ steffen/teaching/grad/partial . pdf. Lecture notes.

Leonhardt, U. (1997): Measuring the Quantum State of Light. (Cambridge University Press, Cambridge).

Ludwig, G. (1983): Foundations of quantum mechanics I. (Springer, New York). Transl. by Carl A. Hein; first publ. in German 1954.

MacColl, L. A., ed. (1957): Applied Probability. (McGraw-Hill, New York).

Månsson, A., Porta Mana, P. G. L., Björk, G. (2006): Numerical Bayesian state assignment for a three-level quantum system. I. Absolute-frequency data; constant and Gaussian-like priors. arXiv: quant - ph/0612105.

Marlow, A. R., ed. (1978): Mathematical Foundations of Quantum Theory. (Academic Press, New York).

Martin-Löf, P. (1974): Repetitive structures and the relation between canonical and microcanonical distributions in statistics and statistical mechanics. In: Barndorff-Nielsen, Blæsild, Schou (1974), 271-294. With discussion.

Mielnik, B. (1968): Geometry of quantum states. Commun. Math. Phys. 9 $^{1}, 55^{-80}$. 
Mielnik, B. (1969): Theory of filters. Commun. Math. Phys. 15 ${ }^{1}, \mathbf{1 - 4 6 .}$

Nielsen, M. A., Chuang, I. L. (2010): Quantum Computation and Quantum Information, 10th anniversary ed. (Cambridge University Press, Cambridge). First publ. 2000.

Owen, D. R. (1984): A First Course in the Mathematical Foundations of Thermodynamics. (Springer, New York).

Peres, A., Terno, D. R. (1998): Convex probability domain of generalized quantum measurements. J. Phys. A $3^{3^{8}}$, L671-L675.

Pitman, E. J. G. (1936): Sufficient statistics and intrinsic accuracy. Math. Proc. Camb. Phil. Soc. $32^{4}, 567-579$.

Pólya, G. (1949): Preliminary remarks on a logic of plausible inference. Dialectica $3^{1-2}, 28-35$.

Porta Mana, P. G. L. (2003): Why can states and measurement outcomes be represented as vectors? arXiv : quant - ph/0305117.

- (2004a): Probability tables. In: Khrennikov (2004), 387-401. Rev. version at arXiv: quant - ph/0403084.

- (2004b): Consistency of the Shannon entropy in quantum experiments. Phys. Rev. A $\mathbf{6 9}^{6}$, 062108. Rev. version at arXiv : quant - ph/0302049.

Porta Mana, P. G. L., Månsson, A., Björk, G. (2006): 'Plausibilities of plausibilities': an approach through circumstances. Being part I of "From 'plausibilities of plausibilities' to state-assignment methods". arXiv: quant - ph/0607111.

Ramsey, F. P. (1926): Truth and probability. In: Ramsey (1950), 156-198. Repr. in Kyburg, Smokler (1980), pp. 23-52. Written 1926.

- (1950): The Foundations of Mathematics: and other Logical Essays. (Routledge \& Kegan Paul, London). Ed. by R. B. Braithwaite. First publ. 1931.

Randall, C. H., Foulis, D. J. (1973): Operational statistics. II. Manuals of operations and their logics. J. Math. Phys. 14 ${ }^{10}, 1472-1480$. See also Foulis, Randall (1972).

- (1979): The operational approach to quantum mechanics. In: Hooker (1979), 167-201.

Ravon, T., Vaidman, L. (2007): The three-box paradox revisited. J. Phys. A $40^{11}, 2873-2882$. See Kirkpatrick (2003b). Unfortunately the arguments of this work are marred by vagueness and contradictions. A reply is given in Kirkpatrick (2007).

Regazzini, E. (1996): De Finetti's reconstruction of the Bayes-Laplace paradigm. Erkenntnis $45^{2}$, 159-176.

Ressel, P. (1985): De Finetti-type theorems: an analytical approach. Ann. Prob. 133 , 898-922. First publ. 1983 as a technical report, https://statistics. stanford. edu/research /de-finetti-type-theorems - analytical-approach.

Schrader, R., Seiler, R., Uhlenbrock, D. A., eds. (1982): Mathematical Problems in Theoretical Physics. (Springer, Berlin).

Segal, I. E. (1959): The mathematical meaning of operationalism in quantum mechanics. In: Henkin, Suppes, Tarski (1959), 341-352.

Shakespeare, W. (1623): As You Like It. Written before 160o. Repr. in Shakespeare (2005).

- (2005): The Complete Works, 2nd ed. (Oxford University Press, Oxford). Ed. by Stanley Wells, Gary Taylor, John Jowett, William Montgomery. First publ. 1986.

Terenin, A., Draper, D. (2017): Cox's theorem and the Jaynesian interpretation of probability. arXiv: 1507.06597. First publ. 2015.

Truesdell III, C. A. (1984): Rational Thermodynamics, 2nd ed. (Springer, New York). First publ. 1969.

van Enk, S. J., Fuchs, C. A. (2002): Quantum state of an ideal propagating laser field. Phys. Rev. Lett. 88, 027902. 
Wigner, E. P. (1959): Group Theory: and Its Application to the Quantum Mechanics of Atomic Spectra, expanded and improved ed. (Academic Press, New York). Transl. by J. J. Griffin. First publ. in German 1931.

Wootters, W. K. (1986): Quantum mechanics without probability amplitudes. Found. Phys. 164, 391-405.

Wright, R. (1978): Spin manuals: Empirical logic talks quantum mechanics. In: Marlow (1978), 177-254.

Ziman, M., Plesch, M., Bužek, V. (2006): Reconstruction of superoperators from incomplete measurements. Found. Phys. $\mathbf{3 6}^{1}, 127-156$. First publ. 2004. 